\title{
Genotypic diversity of multidrug-, quinolone- and extensively drug-resistant Mycobacterium tuberculosis isolates in Thailand
}

Areeya Disratthakit ${ }^{\mathrm{a}}$, Shinji Meada ${ }^{\mathrm{b}}$, Therdsak Prammananan ${ }^{\mathrm{c}}$, Iyarit Thaipisuttikul ${ }^{\mathrm{a}}$, Norio Doi ${ }^{\mathrm{d}}$, Angkana Chaiprasert $\mathrm{a}^{\mathrm{*}^{*}}$

${ }^{a}$ Department of Microbiology, Faculty of Medicine Siriraj Hospital, Mahidol University, Bangkok 10700, Thailand

${ }^{\mathrm{b}}$ Department of Mycobacterium Reference and Research, Research Institute of Tuberculosis, Japan Anti-Tuberculosis Association, Tokyo 204-8533, Japan

${ }^{\mathrm{c}}$ National Center for Genetic Engineering and Biotechnology, National Science and Technology Development Agency, Ministry of Science and Technology, Pathum Thani 12120, Thailand

${ }^{\mathrm{d}}$ Department of Pathophysiology and Host Defense, Research Institute of Tuberculosis, Japan Anti-tuberculosis Association, Tokyo 204-8533, Japan

* Corresponding author: Tel.: +662 419 8256; fax: +662 4182094

E-mail address: angkana.cha@mahidol.ac.th. 


\section{Abstract}

Drug-resistant tuberculosis (TB), which includes multidrug-resistant (MDR-TB), quinolone-resistant (QR-TB) and extensively drug-resistant tuberculosis (XDR-TB), is a serious threat to TB control. We aimed to characterize the genotypic diversity of drugresistant TB clinical isolates collected in Thailand to establish whether the emergence of drug-resistant $\mathrm{TB}$ is attributable to transmitted resistance or acquired resistance. We constructed the first molecular phylogeny of MDR-TB $(n=95), Q R-T B(n=69)$ and XDRTB $(n=28)$ in Thailand based on spoligotyping and proposed 24-locus multilocus variable-number of tandem repeat analysis (MLVA). Clustering analysis was performed using the unweighted pair group method with arithmetic mean. Spoligotyping identified the Beijing strain (SIT1) as the most predominant genotype $(\mathrm{n}=139 ; 72.4 \%)$. The discriminatory power of 0.9235 Hunter-Gaston Discrimination Index (HGDI) with the 15-locus variable-number tandem repeats of mycobacterial interspersed repetitive units typing was improved to a 0.9574 HGDI with proposed 24-locus MLVA, thereby resulting in the subdivision of a large cluster of Beijing strains (SIT1) into 17 subclusters. We identified the spread of drug-resistant TB clones caused by three different MLVA types in the Beijing strain (SIT1) and a specific clone of XDR-TB caused by a rare genotype, the Manu-ancestor strain (SIT523). Overall, 49.5\% of all isolates were clustered. These findings suggest that a remarkable transmission of drug-resistant TB occurred in Thailand. The remaining 50\% of drug-resistant TB isolates were unique genotypes, which may have arisen from the individual acquisition of drug resistance. Our results suggest that transmitted and acquired resistance have played an equal role in the emergence of 
drug-resistant TB. Further characterization of whole genome sequences of clonal strains could help to elucidate the mycobacterial genetic factors relevant for drug resistance, transmissibility and virulence.

Keywords: multidrug resistance; extensively drug-resistant; tuberculosis; MLVA; genotypic diversity; Thailand 


\section{Introduction}

Despite improvements in tuberculosis (TB) treatment, with a success rate among all new TB cases reaching $86 \%$, only $48 \%$ of multidrug-resistant tuberculosis (MDR-TB) patients are treated successfully (World Health Organization, 2014). The poor outcome of this treatment contributes to an increase in the mortality rate and leads to a greater risk of developing extensively drug-resistant tuberculosis (XDR-TB), which is considerably more difficult to treat. The World Health Organization classified the burden of MDR-TB and XDR-TB as a public health crisis. In Thailand, MDR-TB accounts for $2 \%$ and $19 \%$ of new TB and retreatment cases, respectively (World Health Organization, 2014). We analyzed data from routine second-line drug susceptibility testing of MDR-TB between 2003 and 2011. During this eight years period, quinolone-resistant tuberculosis (QR-TB), which is one form of pre-XDR-TB, increased from $9 \%$ to $15 \%$ of MDR-TB isolates, while XDR-TB isolates, which are resistant to quinolone and a second-line injectable drug, comprised $4 \%$ of MDR-TB isolates (Chaiprasert et al., 2014). Elucidation of the factors driving the emergence of drug-resistant $\mathrm{TB}$ is required to optimize strategies for TB control.

Epidemiological investigation is the first-line approach in the identification of the emergence of an infectious disease. However, it does not provide sufficient information about the mechanisms of emergence. The emergence of drug-resistant TB is complicated by two potential mechanisms: (i) transmission of drug resistance from a drug-resistant strain (transmitted resistance) and (ii) acquired drug resistance by a drug-susceptible strain (acquired resistance) (Blower et al., 2004; Klopper et al., 2013). Molecular 
epidemiology has been used to clarify the cause of emerging drug-resistant TB in the community. The most notable application was the use of IS6110-based restriction fragment length polymorphism genotyping in the investigation of the emergence of MDR-TB in New York City from 1995 to 2001, which resulted in the discovery of the transmission of a specific strain of the W/Beijing genotype (Frieden et al., 1995; Munsiff et al., 2002).

A number of genotyping methods have been used to provide information concerning the genetic diversity among drug-resistant strains. Currently, multilocus variable-number of tandem repeat analysis (MLVA) is accepted as a standard polymerase chain reaction (PCR)-based method that is used in molecular epidemiology studies of TB, because it facilitates rapid analysis and provides digital data, which enable the comparison of data between laboratories (Mazars et al., 2000). MLVA data have been used in combination with classical epidemiologic data to provide detailed information on the relative proportion of acquired resistance versus transmitted resistance based on the genotypic diversity over time (de Beer et al., 2014; Marais et al., 2013; Shamputa et al., 2010).

In general, 15-locus variable-number tandem repeats of mycobacterial interspersed repetitive units (MIRU-VNTR) and 24-locus MIRU-VNTR are standard methods used in routine epidemiologic investigations and phylogenetic analysis, respectively (Supply et al., 2006). However, these techniques have the disadvantage of low discriminatory power between the Beijing family of Mycobacterium tuberculosis strains (Allix-Béguec et al., 2014; Gurjav et al., 2014), which is the most prevalent genotype in Thailand (Faksri et al., 2011; Park et al., 2000; Prodinger et al., 2001; Srilohasin et al., 2014). Hypervariable loci have been proposed to improve the efficiency of subtyping Beijing clusters. In this 
study, we selected nine additional loci, which are described as highly polymorphic loci with allelic diversity among Beijing stains above 0.5 in previous reports (Allix-Béguec et al., 2014; Iwamoto et al., 2007; Murase et al., 2008).

Currently, genotyping of $M$. tuberculosis is not routinely performed in Thailand. Therefore, information about emerging drug-resistant TB is based predominantly on classical epidemiologic data without corresponding data informed by genetics (Jirapongsa et al., 2012; Lapphra et al., 2013; Punnotok et al., 2000). In this study, we aimed to characterize the genotypic diversity of drug-resistant TB clinical isolates (including MDR-TB, QR-TB and XDR-TB) collected in Thailand to establish whether the ongoing problem of drug-resistant TB is caused by the transmission of predominant clones or the emergence of acquired resistance. Spoligotyping, a simple and rapid method for detecting polymorphisms within the direct repeat locus, was used as a first-line genotyping tool. Furthermore, a proposed 24-locus MLVA was used for further subtyping of drugresistant $M$. tuberculosis isolates. The discriminatory power of proposed 24-locus MLVA was evaluated in comparison with the standard 15-locus MIRU-VNTR to determine the effect of the nine additional loci on the subtyping of Beijing strains. 


\section{Materials and Methods}

\subsection{Mycobacteria and drug susceptibility testing}

Two hundred and ten M. tuberculosis isolates from 196 TB patients were retrieved from stock cultures of clinical isolates deposited at the Drug-Resistant Tuberculosis Research Fund, Department of Microbiology, Faculty of Medicine Siriraj Hospital, Mahidol University from December 2003 to March 2013. The mycobacterial collection consisted of isolates from thirty provinces in all six geographical regions of Thailand. All isolates were obtained from decontaminated sputum cultured on Löwenstein-Jensen medium, followed by drug susceptibility testing using the proportion method on Middlebrook 7H10 agar quadrant plates. In total, 98 MDR-TB isolates, 82 QR-TB isolates and 30 XDR-TB isolates were successfully retrieved. The stock cultures were stored in Middlebrook $7 \mathrm{H} 9$ media with $15 \%$ glycerol at $-20^{\circ} \mathrm{C}$. The study protocol was approved by the Ethical and Scientific Committees of the Faculty of Medicine Siriraj Hospital, Mahidol University (EC No. Si 029/2557).

\subsection{Genotyping of M. tuberculosis}

\subsubsection{Spoligotyping}

Genomic DNA was obtained by extraction using the cetyl-trimethyl-ammonium bromide-sodium chloride method, as previously described (van Soolingen et al., 1991). The whole sequence of the direct repeat (DR) region, including all spacers between the DR loci, was amplified by simplex PCR. The PCR amplicons were then used to perform 
spoligotyping with a commercially available kit (Ocimum Biosolutions, India) as previously described (Kamerbeek et al., 1997). The binary spoligotype pattern was converted into a 15-digit octal code, which was later used to query the SITVIT WEB (http://www.pasteur-guadeloupe.fr:8081/SITVIT_ONLINE/) proprietary database of the Pasteur Institute of Guadeloupe for classification into clade and spoligotype international type (SIT) (Demay et al., 2012).

\subsubsection{Multilocus variable-number of tandem repeat analysis}

A set of standard 15-locus MIRU-VNTR (Supply et al., 2006), a consensus 4-locus set of hypervariable loci (1982, 3232, 3820 and 4120) (Allix-Béguec et al., 2014), a promising 4-locus set for subtyping Beijing family (2074, 2372, 3155 and 3336) (Murase et al., 2008) and a highly polymorphic locus (2163a) (Iwamoto et al., 2007) were amplified by multiplex PCR or a simplex PCR using 5 'fluorescently labeled primers as shown in Supplementary Table S1. The multiplex PCR and simplex PCR master mixes were prepared as summarized in Supplementary Table S2 and S3, respectively. PCR reactions were performed under the following conditions: initial denaturation at $94^{\circ} \mathrm{C}$ for $5 \mathrm{~min} ; 35$ cycles of denaturation at $94^{\circ} \mathrm{C}$ for $30 \mathrm{~s}$, annealing at $63^{\circ} \mathrm{C}$ for $30 \mathrm{~s}$ and extension at $72^{\circ} \mathrm{C}$ for $1.5 \mathrm{~min}$, with a final extension at $72^{\circ} \mathrm{C}$ for $7 \mathrm{~min}$. The size of the amplified PCR products was determined by capillary electrophoresis on a 3130 genetic analyzer or a 3500 genetic analyzer (Applied Biosystems, USA). The copy number of each locus was automatically estimated using GeneMapper ${ }^{\circledR}$ software v.5 (Applied Biosystems, USA). When an ambiguous peak or no peak was observed, the sample was subjected to re-analysis by simplex PCR with unlabelled primers. In this case, the size of 
the PCR amplicon was determined by $2 \%$ agarose gel electrophoresis or capillary electrophoresis (SV1210 Microchip electrophoresis system, Hitachi High-Technologies Corporation, Japan), and the copy number of each locus was calculated manually.

\subsection{Discriminatory power of genotypic methods and clustering analysis}

The allelic diversity of each locus and the discriminatory power of the different genotypic methods were evaluated based on the $h$-value (Selander et al., 1986) and Hunter-Gaston Discriminatory Index (HGDI) (Hunter et al., 1988), respectively. For clustering analysis, the unweighted pair group method with arithmetic mean (UPGMA) was used to construct a rooted tree based on the categorical coefficients by Ridom MLVA Compare 1.05 (Ridom GmbH, Münster, Germany). A cluster was defined as two or more M. tuberculosis isolates with an identical genotype. 


\section{Results}

\subsection{Discriminatory power of genotypic methods}

Sequential isolates with identical MLVA patterns that were taken from a single patient were counted as only one isolate for further analysis. Three isolates were excluded due to a lack of amplification of loci 2163a, 2163b and 2165, and one isolate was excluded due to mixed genotypes. Consequently, a total of 192 samples were used to determine the discriminatory power of the different genotypic methods. Spoligotyping had the lowest ability to differentiate $M$. tuberculosis genotype in our studied isolates. A standard 15-locus MIRU-VNTR offered a higher discriminatory power of 0.9235 HGDI among all isolates and 0.8683 HGDI among Beijing stains. A better resolution was obtained using 24-locus MLVA; as a result, discriminatory power among all isolates and Beijing strains were improved to 0.9574 HGDI and 0.9245 HGDI, respectively. The highest discriminatory power of 0.9577 HGDI among all isolates was provided by a combination of 24-locus MLVA and spoligotyping. A set of nine additional loci was superior to discriminate non-Beijing strains (0.9690 HGDI) than Beijing strains (0.9194 HGDI). Despite this, the maximum discriminatory power of 0.9834 HGDI among nonBeijing strains could be achieved with the standarad15-locus MIRU-VNTR (Table 1). As summarized in Table 2, five of the nine additional loci (loci 1982, 2163a, 3232, 3820 and 4120) showed high allelic diversity $(h$-value $>0.5)$ for the Beijing strains, although only one of the 15 standard loci (locus 2163b) showed high variability among the Beijing strains. 


\subsection{Spoligotyping-based clustering of isolates}

Using spoligotyping, 174 isolates formed nine clusters, while the remaining 18 isolates had unique genotypes, thus resulting in a total of 27 spoligotypes. A comparison of the spoligotypes from the three different types of resistant strains revealed the marked differences in genotypic diversity among these three types (Table 3 ). In addition to the predominance of Beijing strain (SIT1) clone in MDR-TB (75.8\%), QR-TB (73.9\%) and XDR-TB (57.1\%), the Manu-ancestor (SIT523) clone was the second most common genotype, accounting for 4.3\% of QR-TB and an overrepresentation in XDR-TB at $39.2 \%$, although it was absent from the MDR-TB group. In contrast, the T1 clade (SIT53) was the second most common clone in MDR-TB (4.2\%). In addition, most of the unique spoligotypes that were found in MDR-TB were not found in QR-TB or XDR-TB. Three MDR-TB isolates were defined as orphan. Two of them exist in SITVIT WEB database as single isolates belonging to EAI5 and EAI6_BGD genotype. The remaining one did not match any existing spoligotype in the database.

\subsection{MLVA-based clustering of isolates}

Using 24-locus MLVA, the 192 isolates were divided into 115 MLVA types. A total of 95 isolates (49.5\%) formed 18 different clusters, which ranged in size from two isolates to 36 isolates (Table 1). The remaining 97 isolates (50.5\%) had unique genotypes. Three major MLVA clusters of the Beijing family (SIT1) were identified: cluster $4(\mathrm{n}=37), 5(\mathrm{n}=8)$ and $11(\mathrm{n}=10)$ and were defined as predominant clones because these clusters were found in all three resistance types, were isolated from all regions of Thailand over the course of the study period and comprised $28.6 \%$ of all 
isolates in this study. Intriguingly, the fourth largest cluster (cluster 18; $n=6$ ) was specific to only XDR-TB with a distinct MLVA pattern typical of a Manu-ancestor (SIT523) clone (Table 4). Consequently, the isolates that were clustered among the three types of drug-resistant TB were categorized as belonging to two cluster types: (i) predominant clones and (ii) minor clusters. The relative proportion of predominant clones decreased from $32.6 \%(n=31 / 95)$ of MDR-TB to $21.4 \%(n=6 / 28)$ of XDR-TB, while the relative proportion of minor clusters increased from $14.7 \%(n=14 / 95)$ of MDR-TB to $28.6 \%(n=8 / 28)$ of XDR-TB. Approximately $50 \%$ of all types of drug-resistant TB were singletons (unique genotypes) (Table S4).

\subsection{Clonal spread of MDR-TB and XDR-TB in Western Thailand}

The combination of genotypic data and geographical information highlighted two instances of MDR-TB and XDR-TB spread in the vicinity of Kanchanaburi Province in Western Thailand. First, the largest clonal complex of MDR-TB, which comprised 15 isolates from a predominant clone, cluster 4, and two single locus variants, were detected sporadically during the period from 2006 to 2010 (Fig. 2A). Second, the clonal spread of XDR-TB with the specific MLVA type (Cluster 18) of Manu-ancestor (SIT523) was identified during the period from 2007 to 2012. Clonal complexes of this specific MLVA type and its single locus variants accounted for $25.0 \%$ (7 of 28 isolates) of the XDR-TB cases in this study (Fig. 2B). The partial map of Western part of Thailand is shown in figure $2 \mathrm{C}$. 


\section{Discussion}

The emergence of drug-resistant TB, including MDR-TB, QR-TB and XDR-TB, is continuously reported in Thailand (Chaiprasert et al., 2014; World Health Organization, 2014). Elucidation of the mechanisms underlying this problem is required for the development of appropriate control strategies. In this study, spoligotyping revealed the predominance of the Beijing genotype (SIT1), which accounted for the largest cluster (72.6\%) among all the isolates. However, such a high percentage does not necessarily reflect an actual transmission event because of the poor discriminatory power of spoligotyping among Beijing strains, as shown in Table 1.

The present study confirmed that five of the additional loci (1982, 2163a, 3232, 3820 and 4120) contributed significantly to the subtyping of both Beijing and nonBeijing isolates as previous report (Allix-Béguec et al., 2014; Iwamoto et al., 2007; Murase et al., 2008). Although locus 2163a was excluded from the previous report due to amplification failure or an unreliably large amplification product, we included this locus because it represented characteristic high copy number of 18 copies (PCR product, approximately $1,400 \mathrm{bp}$ ) in two major clonal complexes of transmitted strains that caused clonal expansion of MDR-TB and XDR-TB. The remaining four loci $(2074,2372,3155$ and 3336) had low allelic diversity among the Beijing isolates, which is inconsistent with those of a previous study examining Japanese isolates (Murase et al., 2008). These discrepancies may be due to differences in the proportion of ancestral and modern Beijing strains. The percentage of ancestral Beijing strains is high in Japan (Iwamoto et al., 2009), whereas modern Beijing strains are more predominant in Thailand (Faksri et al., 
2011; Srilohasin et al., 2014). This hypothesis is supported by Luo et al. (2014), who found lower variability in VNTRs 2074 and 2372 from the ancestral Beijing strains than in the modern Beijing strains from China.

In this study, MLVA revealed a moderate level of genotypic diversity, which implicated composite mechanisms of resistance, including transmitted and acquired resistance, as a potential cause for the emergence of drug-resistant TB. In the present study, we have identified three predominant clones of the Beijing genotype (SIT1) as major causes of TB transmission. Many studies that examined the association between mycobacterial genetic background and transmission rate have demonstrated a higher transmission rate among Beijing strains than non-Beijing strains (Sun et al., 2007; van der Spuy et al., 2009; Yang et al., 2012). Despite this, variety exists among the Beijing strains. Hanekom et al. described differences among different Beijing sublineages in their ability to transmit and cause disease (Hanekom et al., 2007). This variation might explain why in this study we could distinguish the three different predominant clones of the Beijing strain (SIT1). In addition, we have observed that the predominant clones represented all three types of resistance. These data indicated that amplification of resistance to second-line drugs in MDR-TB may occur in those clones, thus leading to the development of QR-TB and XDR-TB from MDR-TB.

Surprisingly, we found that the fourth largest MLVA cluster of a rare SIT523 spoligotype accounted for $3.13 \%$ of all isolates in our study, whereas this spoligotype has been reported in only 27 of 58,180 global isolates $(0.05 \%)$ and two of 307 Thai isolates $(0.65 \%)$ in the SITVIT WEB database (Demay et al., 2012). The Manu-ancestor (SIT523) strain is considered to be a part of the Indo-Oceanic (lineage 1) (Shabbeer et al., 
2012). However, the minimum spanning tree based on MLVA data and whole genome SNP analysis demonstrated the connection between SIT523 and East Asian (lineage 2; includes Beijing family) (Blouin et al., 2012). Similarly, the minimum spanning tree created using MLVA profiles in our study also demonstrated that the SIT523 cluster was connected to cluster 4, the largest predominant clone of the Beijing genotype (SIT1) (data not shown), which might explain why SIT523 acquired efficient transmissibility. Interestingly, this cluster was only specific to XDR-TB. The dynamics of drug-resistant TB transmission vary depending on resistance-conferring mutations. Some specific mutations cause no fitness defect or compensate for fitness cost, for instance, $r p o B$ S531L, katG S315T and compensatory mutations in rpoA and rpoC. These mutations are found to be associated with successful transmission (Casali et al., 2014; Gagneux, 2009). A distinct SIT523 strain may gain such mutations in its genome. Hence, our study emphasizes the problem of clonal expansion of a certain strain and the need for further examination of the transmissibility of this strain, which may contribute to the possibility of clonal expansion.

Approximately $50 \%$ of the isolates from each resistance group were unique genotypes (Fig. 1), which suggests that drug resistance developed in individual patients over the study period. There are limitations on interpretation of our results. The small and unequal sample size in each resistance group may affect the clustering analysis. In addition, geographic data is only available for predicting clonal expansions in this study; therefore, additional epidemiological and demographic data are needed to clarify the transmission. Furthermore, an adequate treatment history or investigation of drug susceptibility of sequential isolates for each patient is required to more precisely define 
cases of acquired resistance. Despite these limitations, the selection of mycobacterial isolates was based on phenotypic resistance data only, without prior knowledge of the strain types; thus, the genotypic diversity observed is likely to reflect the true diversity among M. tuberculosis isolates in this study.

To our knowledge, this study provides the first insight into the genotypic diversity of drug-resistant TB in Thailand. Our results show the usefulness of genotypic diversity as a supportive tool for understanding the causes of emergence drug-resistant TB over study period. Firstly, the clonal spread of MDR-TB and XDR-TB provided the genetic evidence of previous MDR-TB community outbreak (Jirapongsa et al., 2012) and revealed a hidden spreading event of XDR-TB, respectively. Secondly, three major clusters among all resistance groups suggested the spread of predominant clones and amplification of resistance. Finally, a moderate number of unique genotypes hinted at possibility of acquired resistance as another factor driving the emergence of drugresistant TB. The results of this study emphasize the need to strengthen TB control strategies and to adopt appropriate treatment regimens to prevent the further development of drug resistance. Additionally, a comprehensive study of whole genome sequences is required for understanding of the genetic factors that are relevant to the drug resistance, transmissibility and virulence of clonal strains. 


\section{Acknowledgements}

Financial support for this study was obtained from the Thailand Research Fund through the Royal Golden Jubilee Ph.D. Program (Grant No. PHD/0328/2552) to Areeya Disratthakit (a student) and Angkana Chaipreaert (advisor). Financial support for the laboratories was also obtained from the Drug Resistant Tuberculosis Research Fund Siriraj Foundation, Research Development Fund, Faculty of Medicine Siriraj Hospital, Mahidol University and Japan Science and Technology/National Science and Technology Development Agency, Thailand (JST/NSTDA, Grant No. P-12-01777). AC is supported by the Chalermprakiat grant from the Faculty of Medicine Siriraj Hospital, Mahidol University.

We would like to thank the Research Institute of Tuberculosis, Japan AntiTuberculosis Association for research support to perform the experiments for 2 months.

This work is dedicated to the late HRH Princess Galyanivadhana KromLaung Narathiwas Rajnakarindth, the patron of the Drug Resistant Tuberculosis Fund Siriraj Foundation on the occasion of HRH's 91st Birthday. 


\section{References}

Allix-Béguec, C., Wahl, C., Hanekom, M., Nikolayevskyy, V., Drobniewski, F., Maeda, S., Campos-Herrero, I., Mokrousov, I., Niemann, S., Kontsevaya, I., Rastogi, N., Samper, S., Sng, L., Warren, R.M., Supply, P., 2014. Proposal of a consensus set of hypervariable mycobacterial interspersed repetitive-unit-variable-number tandemrepeat loci for subtyping of Mycobacterium tuberculosis Beijing isolates. J. Clin. Microbiol. 52 (1), 164-172.

Blouin, Y., Hauck, Y., Soler, C., Fabre, M., Vong, R., Dehan, C., Cazajous, G., Massoure, P., Kraemer, P., Jenkins, A., Garnotel, E., Pourcel, C., Vergnaud, G., 2012. Significance of the identification in the Horn of Africa of an exceptionally deep branching Mycobacterium tuberculosis clade. PLoS ONE. 7 (12), e52841.

Blower, S.M., Chou, T., 2004. Modeling the emergence of the 'hot zones': tuberculosis and the amplification dynamics of drug resistance. Nat. Med. 10 (10), 1111-1116.

Casali, N., Nikolayevskyy, V., Balabanova, Y., Harris, S.R., Ignatyeva, O., Kontsevaya, I., Corander, J., Bryant, J., Parkhill, J., Nejentsev, S., Horstmann, R.D., Brown, T., Drobniewski, F., 2014. Evolution and transmission of drug-resistant tuberculosis in a Russian population. Nat. Genet. 46 (3), 279-286.

Chaiprasert, A., Srimuang, S., Tingtoy, N., Makhao, N., Sirirudeeporn, P., Tomnongdee, N., Theankeaw, O., Charoensook, S., Leechawengwongs, M., Prammananan, T., 2014. Second-line drug susceptibilities of multidrug-resistant tuberculosis strains isolated in Thailand: an update. Int. J. Tuberc. Lung Dis. 18 (8), 961-963.

De Beer, J.L., Ködmön, C., van der Werf, M.J., van Ingen, J., van Soolingen, D., the ECDC MDR-TB molecular surveillance project participants, 2014. Molecular 
surveillance of multi- and extensively drug-resistant tuberculosis transmission in the European Union from 2003 to 2011. Euro Surveill. 19 (11), pii=20742. Available from: $<$ http://www.eurosurveillance.org/ViewArticle.aspx?ArticleId=20742>

Demay, C., Liens, B., Burguière, T., Hill V., Couvin, D., Millet, J., Mokrousov, I., Sola, C., Zozio, T., Rastogi, N., 2012. SITVITWEB - A publicly available international multimarker database for studying Mycobacterium tuberculosis genetic diversity and molecular epidemiology. Infect. Genet. Evol. 12, 755-766.

Faksri, K., Drobniewski, F., Nikolayevskyy, V., Brown, T., Prammananan, T., Palittapongarnpim, P., Prayoonwiwat, N., Chaiprasert, A., 2011. Epidemiological trends and clinical comparisons of Mycobacterium tuberculosis lineages in Thai TB meningitis. Tuberculosis. 91, 594-600.

Frieden, T.R., Fujiwara, P.I., Washko, R.M., Hamburg, M.A., 1995. Tuberculosis in New York City - turning the tide. N. Engl. J. Med. 333 (4), 229-233.

Gagneux, S., 2009. Fitness cost of drug resistance in Mycobacterium tuberculosis. Clin. Microbiol. Infect. 15 (Suppl. 1), 66-68.

Gurjav, U., Jelfs, P., MaCallum, N., Marais, B.J., Sintchenko, V., 2014. Temporal dynamics of Mycobacterium tuberculosis genotypes in New South Wales, Australia. BMC Infect. Dis. 14, 455.

Hanekom, M., van der Spuy, G.D., Streicher, E., Ndabambi, S.L., McEvoy, C.R.E., Kidd, M., Beyers, N., Victor, T.C., van Helden, P.D., Warren, R.M., 2007. A recently evolved sublineage of the Mycobacterium tuberculosis Beijing strain family is associated with and increased ability to spread and cause disease. J. Clin. Microbiol. $45(5), 1483-1490$. 
Hunter, P.R., Gaston, M.A., 1988. Numerical index of the discriminatory ability of typing systems: an application of Simpson's index of diversity. J. Clin. Microbiol. 26 (11), $2465-2466$.

Iwamoto, T., Yoshida, S., Suzuki, K., Tomita, M., Fujiyama, R., Tanaka, N., Kawakami, Y., Ito, M., 2007. Hypervariable loci that enhance the discriminatory ability of newly proposed 15-loci and 24-loci variable-number tandem repeat typing method on Mycobacterium tuberculosis strains predominated by the Beijing family. FEMS Microbiol. Lett. 270, 67-74.

Iwamoto, T., Fujiyama, R., Yoshida, S., Wada, T., Shirai, C., Kawakami, Y., 2009. Population structure dynamics of Mycobacterium tuberculosis Beijing strains during past decades in Japan. J. Clin. Microbiol. 47 (10), 3340-3343.

Jiraphongsa, C., Wangteeraprasert, T., Henpraserttae. N., Sanguanwongse, N., Panya, L., Sukkasitvanichkul, J., Pittayawonganon, C., 2011. Community outbreak of multidrug resistant tuberculosis, Kanchanaburi province, Thailand on 2002-June 2010. J. Preventive Med. Assoc. Thai. 1 (3), 261-271.

Kamerbeek, J., Schouls, L., Kolk, A., van Agterveld, M., van Soolingen, D., Kuijper, S., Bunschoten, A., Molhuizen, H., Shaw, R., Goyal, M., van Embden, J., 1997. Simultaneous detection and strain differentiation of Mycobacterium tuberculosis for diagnosis and epidemiology. J. Clin. Microbiol. 35 (4), 907-914.

Klopper, M., Warren, R.M., Hayes, C., van Pittius, N.C.G., Streicher, E.M., Müller, B., Sirgel, F.A., Chabula-Nxiweni, M., Hoosain, E., Coetzee, G., van Helden, P.D., Victor, T.C., Trollip, A.P., 2013. Emergence and spread of extensively and totally drug-resistant tuberculosis, South Africa. Emerg. Infect. Dis. 19 (3), 449-455. 
Lapphra, K., Sutthipong, C., Foongladda, S., Vanprapar, N., Phongsamart, W., Wittawatmongkol, O., Saksawad, R., Chokephaibulkit, K., 2013. Drug-resistant tuberculosis in children in Thailand. Int. J. Tuberc. Lung Dis. 17 (10), 1279-1284.

Luo, T., Yang, C., Gagneux, S., Gicquel, B., Mei, J., Gao, Q., 2012. Combination of single nucleotide polymorphism and variable-number tandem repeats for genotyping a homogenous population of Mycobacterium tuberculosis Beijing strains in China. J. Clin. Microbiol. 50 (3), 633-639.

Luo, T., Yang, C., Pang, Y., Zhao, Y., Mei, J., Gao, Q., 2014. Development of a hierarchical variable-number tandem repeat typing scheme for Mycobacterium tuberculosis in China. PLoS ONE. 9 (2), e89726.

Marais, B.J., Mlambo, C.K., Rastogi, N., Zozio, T., Duse, A.G., Victor, T.C., Marais, E., Warren, R.M., 2013. Epidemic spread of multidrug-resistant tuberculosis in Johannesburg, South Africa. J. Clin. Microbiol. 51 (6), 1818-1825.

Mazars, E., Lesjean, S., Banuls, A., Gilbert, M., Vincent, V., Gicquel, B., Tibayrenc, M., Locht, C., Supply, P., 2001. High-resolution minisatellite-based typing as a portable approach to global analysis of Mycobacterium tuberculosis molecular epidemiology. Proc. Natl. Acad. Sci. USA. 98 (4), 1901-1906.

Munsiff, S.S., Bassoff, T., Nivin, B., Li, J., Sharma, A., Bifani, P., Mathema, B., Driscoll, J., Kreiswirth, B.N., 2002. Molecular epidemiology of multidrug-resistant tuberculosis, New York City, 1995-1997. Emerg. Infect. Dis. 8 (11), 1230-1238. Murase, Y., Mitarai, S., Sugawara, I., Kato, S., Maeda, S., 2008. Promising loci of variable numbers of tandem repeats for typing Beijing family Mycobacterium tuberculosis. J. Med. Microbiol. 57, 873-880. 
Park, Y.K., Bai, G.H., Kim, S.J., 2000. Restriction fragment length polymorphism analysis of Mycobacterium tuberculosis isolated from countries in the western pacific region. J. Clin. Microbiol. 38 (1), 191-197.

Prodinger, W.M., Bunyaratvej, P., Prachaktam, R., Pavlic, M., 2001. Mycobcaterium tuberculosis isolates of Beijing genotype in Thailand. Emerg. Infect. Dis. 7 (3), 483484.

Punnotok, J., Shaffer, N., Naiwatanakul, T., Pumprueg, U., Subhannachart, P., Ittiravivongs, A., Chuchotthaworn, C., Ponglertnapagorn, P., Chantharojwong, N., Young, N.L., Limpakarnjanarat, K., Mastro, T.D., 2000. Human immunodeficiency virus-related tuberculosis and primary drug resistance in Bangkok, Thailand. Int. J. Tuberc. Lung Dis. 4 (6), 537-543.

Selander, R.K., Caugant, D.A., Ochman, H., Musser, J.M., Gilmour, M.N., Whittam, T.S., 1986. Methods of multilocus enzyme electrophoresis for bacterial population genetics and systematics. Appl. Environ. Microbiol. 51 (5), 873-884.

Shabbeer, A., Cowan, L.S., Ozcaglar, C., Rastogi, N., Vandenberg, S.L., Yener, B., Bennett, K.P., 2012. TB-Lineage: An online tool for classification and analysis of strains of Mycobacterium tuberculosis complex. Infect. Genet. Evol. 12, 789-797. Shamputa, I.C., Lee, J., Allix-Béguec, C., Cho, E., Lee, J., Rajan, V., Lee, E.G., Min, J.H., Carroll, M.W., Goldfeder, L.C., Kim, J.H., Kang, H.S., Hwang, S., Eum, S., Park, S.K., Lee, H., Supply, P., Cho, S., Via, L.E., Barry III, C.E., 2010. Genetic diversity of Mycobacterium tuberculosis isolates from a tertiary care tuberculosis hospital in South Korea. J. Clin. Microbiol. 48 (2), 387-394. 
Srilohasin, P., Chaiprasert, A., Tokunaga, K., Nishida, N., Prammananan, T., Smittipat, N., Mahasirimongkol, S., Chaiyasirinroje, B., Yanai, H., Palittapongarnpim, P., 2014. Genetic diversity and dynamic distribution of Mycobacterium tuberculosis isolates causing pulmonary and extrapulmonary tuberculosis in Thailand. J. Clin. Microbiol. 52 (12), 4267-4274.

Sun, J.Y., Lee, A.S.G., Wong, S.Y., Heersma, H., Kremer, K., van Soolingen, D., Paton, N.I., 2007. Genotype and phenotype relationships and transmission analysis of drugresistant tuberculosis in Singapore. Int. J. Tuberc. Lung. Dis. 11 (4), 436-442.

Supply, P., Allix, C., Lesjean, S., Cardoso-Oelemann, M., Rüsch-Gerdes, S., Willery, E., Savine, E., de Haas, P., van Deutekom, H., Roring, S., Bifani, P., Kurepina, N., Kreiswirth, B., Sola, C., Rastogi, N., Vatin, V., Gutierrez, M.C., Fauville, M., Niemann, S., Skuce, R., Kremer, K., Locht, C., van Soolingen, D., 2006. Proposal for standardization of optimized mycobacterial interspersed repetitive unit-variablenumber tandem repeat typing of Mycobacterium tuberculosis. J. Clin. Microbiol. 44 (12), 4498-4510.

van der Spuy, G.D., Kremer, K., Ndabambi, S.L., Beyers, N., Dunbar, R., Marais, B.J., van Helden, P.D., Warren, R.M., 2009. Changing Mycobacterium tuberculosis population highlights clade-specific pathogenic characteristics. Tuberculosis. 89, $120-125$.

van Soolingen, D., Hermans, P.W., de Haas, P.E., Soll, D.R., van Embden, J.D., 1991. Occurrence and stability of insertion sequences in Mycobacterium tuberculosis complex strains: evaluation of an insertion sequence-dependent DNA polymorphism as a tool in the epidemiology of tuberculosis. J. Clin. Microbiol. 29(11), 2578-2586. 
World Health Organization, 2014. Global Tuberculosis Report 2014. WHO, Geneva,

Switzerland. Available from: <http://apps.who.int/iris/bitstream/10665/137094/1/ 9789241564809_eng.pdf>

Yang, C., Luo, T., Sun, G., Qiao, K., Sun, G., DeRiemer, K., Mei, J., Qian, G., 2012. Mycobacterium tuberculosis Beijing strains favor transmission but not drug resistance in China. Clin. Infect. Dis. 55 (9), 1179-1187.

Zheng, C., Zhao, Y., Zhu, G., Li, S., Sun, H., Feng, Q., Luo, M., Wu, F., Li, X., Hill, V., Rastogi, N., Sun, Q., 2014. Suitability of IS6110-RFLP and MIRU-VNTR for differentiating spoligotyped drug-resistant Mycobacterium tuberculosis clinical isolates from Sichuan in China. Biomed. Res. Int. 2014, 763204. 


\section{Legends}

Table 1. Discriminatory power of spoligotyping and different multilocus variablenumber of tandem repeat analysis schemes.

Table 2. Allelic diversity of each locus in the 24-locus MLVA scheme.

Table 3. Spoligotypes of multidrug-resistant (MDR), quinolone-resistant (QR) and extensively drug-resistant (XDR) tuberculosis.

Table 4. MLVA-based cluster details of drug-resistant TB isolates from all regions of Thailand from December 2003 to March 2013.

Fig. 1. Proportion of singletons, minor clusters and predominant clones among three different types of resistance. A singleton is a genotype appearing uniquely within the data set. Predominant clones are isolates in clusters 4, 5 and 11, consisting of all three types of drug-resistant TB, and are continuously isolated from all regions of Thailand. Minor clusters are the remaining clustered isolates. The numbers on each bar represent number of isolates in different categories; where $\mathrm{n}$ represent the total number of isolates of multidrug-resistant (MDR), quinolone-resistant (QR) and extensively drug-resistant (XDR) tuberculosis.

Fig. 2. Unweighted pair group method with arithmetic mean tree based on the 24-locus MLVA data of multidrug-resistant TB (A) and extensively drug-resistant TB (B) isolates from the vicinity of Kanchanaburi Province in Western Thailand. Boxes with dashed lines highlight the clonal complexes transmitted in this area. (C) Map of Kanchanaburi Province and vicinities: (1) Kanchanaburi, (2) Ratchaburi, (3) Suphanburi, (4) Bangkok and (5) Myanmar. 


\section{Table 1}

Discriminatory power of spoligotyping and different multilocus variable-number of tandem repeat analysis schemes.

\begin{tabular}{|c|c|c|c|c|c|c|c|}
\hline \multirow{2}{*}{ Methods } & \multirow{2}{*}{$\begin{array}{l}\text { Clustered } \\
\text { types }\end{array}$} & \multirow{2}{*}{$\begin{array}{c}\text { Unique } \\
\text { types }\end{array}$} & \multirow{2}{*}{$\begin{array}{l}\text { Percentage } \\
\text { of clustered } \\
\text { isolates }\end{array}$} & \multirow{2}{*}{$\begin{array}{c}\text { Largest } \\
\text { cluster size } \\
(\%)\end{array}$} & \multicolumn{3}{|c|}{$\begin{array}{l}\text { Hunter-Gaston Discriminatory Index } \\
\text { (HGDI) }\end{array}$} \\
\hline & & & & & $\begin{array}{l}\text { All isolates } \\
(n=192)\end{array}$ & $\begin{array}{c}\text { Beijing } \\
(n=143)\end{array}$ & $\begin{array}{c}\text { Non-Beijing } \\
(n=49)\end{array}$ \\
\hline Spoligotyping & 9 & 18 & $90.1 \%$ & $139(72.4)$ & 0.4782 & 0.0551 & 0.8771 \\
\hline 15-locus MIRU-VNTR & 20 & 71 & $63.0 \%$ & $48(25.0)$ & 0.9235 & 0.8683 & 0.9834 \\
\hline 9 additional loci & 21 & 83 & $56.8 \%$ & $37(19.3)$ & 0.9546 & 0.9194 & 0.9690 \\
\hline $\begin{array}{l}\text { 15-locus MIRU-VNTR } \\
+9 \text { additional loci }\end{array}$ & 18 & 97 & $49.5 \%$ & $37(19.3)$ & 0.9574 & 0.9245 & 0.9834 \\
\hline $\begin{array}{l}\text { Spoligotyping } \\
+15 \text {-locus MIRU-VNTR } \\
+9 \text { additional loci }\end{array}$ & 17 & 98 & $48.4 \%$ & $37(19.3)$ & 0.9577 & 0.9253 & 0.9834 \\
\hline
\end{tabular}


Table 2

Allelic diversity of each locus in the 24-locus MLVA scheme.

\begin{tabular}{|c|c|c|c|c|c|c|c|}
\hline \multirow[b]{2}{*}{ Locus } & \multirow{2}{*}{$\begin{array}{l}\text { Unit } \\
\text { (bp) }\end{array}$} & \multirow{2}{*}{$\begin{array}{c}\text { Copy } \\
\text { no. of } \\
\text { H37Rv }\end{array}$} & \multirow{2}{*}{$\begin{array}{l}\text { No. of } \\
\text { alleles }\end{array}$} & \multirow[b]{2}{*}{ Copy number } & \multicolumn{3}{|c|}{ Allelic diversity $(h)$ for: ${ }^{a}$} \\
\hline & & & & & $\begin{array}{l}\text { All isolates } \\
(\mathrm{n}=192)\end{array}$ & $\begin{array}{c}\text { Beijing } \\
(\mathrm{n}=143)\end{array}$ & $\begin{array}{c}\text { Non-Beijing } \\
(\mathrm{n}=49)\end{array}$ \\
\hline \multicolumn{8}{|c|}{ 15-locus MIRU-VNTR } \\
\hline 2163b & 69 & 5 & 9 & $1-9$ & 0.785 & 0.699 & 0.842 \\
\hline 4052 & 111 & 5 & 10 & $1-10$ & 0.559 & 0.347 & 0.822 \\
\hline 3690 & 58 & 5 & 8 & $1-7,10$ & 0.538 & 0.304 & 0.752 \\
\hline 1955 & 57 & 1 & 10 & $1-6,8-10,18$ & 0.534 & 0.357 & 0.790 \\
\hline 2165 & 75 & 3 & 8 & $2-9$ & 0.518 & 0.248 & 0.799 \\
\hline 4156 & 59 & 3 & 5 & $0-4$ & 0.505 & 0.287 & 0.468 \\
\hline 0424 & 51 & 2 & 7 & $1-6,9$ & 0.476 & 0.297 & 0.664 \\
\hline 0960 & 53 & 3 & 6 & $2-5,8,10$ & 0.456 & 0.297 & 0.664 \\
\hline 2996 & 51 & 3 & 8 & $1-3,5-8$ & 0.439 & 0.172 & 0.750 \\
\hline 0580 & 53 & $2.5^{b}$ & 11 & $0-9$ & 0.387 & 0.221 & 0.697 \\
\hline 2401 & 58 & 2 & 4 & $1-2,4-5$ & 0.366 & 0.041 & 0.280 \\
\hline 1644 & 53 & 2 & 4 & $1-4$ & 0.360 & 0.054 & 0.598 \\
\hline 3192 & 53 & 3 & 7 & $2-8$ & 0.336 & 0.081 & 0.710 \\
\hline 0802 & 54 & 1 & 4 & $1-4$ & 0.312 & 0.243 & 0.458 \\
\hline 0577 & 58 & 4 & 4 & $2-5$ & 0.041 & 0.000 & 0.155 \\
\hline \multicolumn{8}{|c|}{9 additional loci } \\
\hline 3820 & 57 & 3 & 14 & $1,3-5,7-12,14-17$ & 0.831 & 0.765 & 0.863 \\
\hline $2163 a$ & 69 & 2 & 11 & $2,4-12,18$ & 0.791 & 0.741 & 0.856 \\
\hline 3232 & 56 & 4 & 18 & $1,4-20$ & 0.779 & 0.639 & 0.788 \\
\hline 4120 & 57 & 2 & 11 & $2-6,8-13$ & 0.734 & 0.574 & 0.725 \\
\hline 1982 & 78 & 5 & 12 & $0,2-12$ & 0.685 & 0.524 & 0.774 \\
\hline 2372 & 57 & 2 & 4 & $1-4$ & 0.414 & 0.206 & 0.574 \\
\hline 3336 & 59 & 8 & 9 & $4-12$ & 0.391 & 0.172 & 0.742 \\
\hline 2074 & 56 & 4 & 4 & $1-4$ & 0.228 & 0.028 & 0.582 \\
\hline 3155 & 54 & 4 & 5 & $2-6$ & 0.193 & 0.146 & 0.319 \\
\hline
\end{tabular}

${ }^{a}$ Data was sorted in descending order of allelic diversity.

${ }^{\mathrm{b}}$ VNTR0580 of M. tuberculosis H37Rv possesses Type I repeat unit (77 bp), while most clinical isolates possess the Type II repeat unit (53 bp). 


\section{Table 3}

Spoligotypes of multidrug-resistant (MDR), quinolone-resistant (QR) and extensively drugresistant (XDR) tuberculosis.

\begin{tabular}{|c|c|c|c|c|c|}
\hline & \multirow{2}{*}{ SIT $^{\mathrm{a}}$} & \multirow{2}{*}{ Clades } & \multicolumn{3}{|c|}{ No. of isolates (\%) } \\
\hline & & & MDR-TB (n = 95) & QR-TB (n = 69) & XDR-TB $(n=28)$ \\
\hline \multirow{9}{*}{$\begin{array}{c}\text { Clustered } \\
\text { Spoligotypes }\end{array}$} & 1 & Beijing & $72(75.8)$ & $51(73.9)$ & $16(57.1)$ \\
\hline & 48 & EAI1_SOM & 0 & $3(4.3)$ & 0 \\
\hline & 50 & H3 & $2(2.1)$ & 0 & 0 \\
\hline & 53 & $\mathrm{~T} 1$ & $4(4.2)$ & 0 & 0 \\
\hline & 236 & EAI5 & $2(2.1)$ & $2(2.9)$ & 0 \\
\hline & 246 & Unknown & $1(1.1)$ & $2(2.9)$ & 0 \\
\hline & 523 & Manu_ancestor & 0 & $3(4.3)$ & $11(39.3)$ \\
\hline & 591 & EAI6_BGD & $2(2.1)$ & 0 & 0 \\
\hline & 941 & Beijing & $3(3.2)$ & 0 & 0 \\
\hline \multirow{18}{*}{$\begin{array}{c}\text { Unique } \\
\text { Spoligotypes }\end{array}$} & 35 & H3 & 0 & $1(1.4)$ & 0 \\
\hline & 89 & EAI2_nonthaburi & $1(1.1)$ & 0 & 0 \\
\hline & 255 & Beijing & 0 & $1(1.4)$ & 0 \\
\hline & 256 & EAI5 & 0 & $1(1.4)$ & 0 \\
\hline & 469 & LAM1 & 0 & 0 & $1(3.6)$ \\
\hline & 497 & EAI5 & $1(1.1)$ & 0 & 0 \\
\hline & 735 & EAI1_SOM & 0 & $1(1.4)$ & 0 \\
\hline & 939 & EAI5 & 0 & $1(1.4)$ & 0 \\
\hline & 942 & $\mathrm{~T} 2$ & 0 & $1(1.4)$ & 0 \\
\hline & 943 & Ambiguous T2-T5 & $1(1.1)$ & 0 & 0 \\
\hline & 955 & Unknown & $1(1.1)$ & 0 & 0 \\
\hline & 962 & EAI5 & 0 & $1(1.4)$ & 0 \\
\hline & 1364 & Beijing & $1(1.1)$ & 0 & 0 \\
\hline & 1378 & Manu3 & 0 & $1(1.4)$ & 0 \\
\hline & 2351 & EAI2_Manila & $1(1.1)$ & 0 & 0 \\
\hline & Orphan & EAI5 & $1(1.1)$ & 0 & 0 \\
\hline & Orphan & EAI6_BGD & $1(1.1)$ & 0 & 0 \\
\hline & Orphan & - & $1(1.1)$ & 0 & 0 \\
\hline
\end{tabular}

a SIT: Spoligotype International Type from SITVITWEB 


\section{Table 4}

MLVA-based cluster details of drug-resistant TB isolates from all regions of Thailand from December 2003 to March 2013.

\begin{tabular}{|c|c|c|c|c|c|c|}
\hline $\begin{array}{c}\text { Cluster } \\
\text { no. }\end{array}$ & Spoligotype & MLVA patterns $^{\mathrm{a}}$ & $\begin{array}{c}\text { No. of } \\
\text { isolates }\end{array}$ & Type of resistance & Origin of isolates & Year of isolation \\
\hline 1 & Beijing (SIT1) & 4423334744753A4A3544F7CC & 2 & MDR & Eastern, Central & 2009,2010 \\
\hline 2 & Beijing (SIT1) & 44233356447538283834D7BA & 4 & MDR & Northeastern, Southern & 2007,2012 \\
\hline 3 & Beijing (SIT1) & 44233356447538283834G7EA & 3 & MDR, QR & Northeastern, Central & 2009 \\
\hline 4 & Beijing (SIT1) & 44233354447538283I34E79A & 37 & $\mathrm{MDR}, \mathrm{QR}, \mathrm{XDR}$ & All regions & 2004-2012 \\
\hline 5 & Beijing (SIT1) & $44233354447538283 \mathrm{I} 34 \mathrm{E} 799$ & 8 & $\mathrm{MDR}, \mathrm{QR}, \mathrm{XDR}$ & Northern, Eastern, Central, Western, Southern & $2005,2007-2010$ \\
\hline 6 & Beijing (SIT1) & 34233356447539083834E7BA & 4 & MDR, QR & Northern, Northeastern, Central & $2005,2007,2012,2013$ \\
\hline 7 & Beijing (SIT1) & 44223354447538283834F9CD & 2 & MDR, QR & Western, Central & 2007,2010 \\
\hline 8 & Beijing (SIT1) & 44223335447537263834F7EA & 2 & QR & Eastern, Central & 2006 \\
\hline 9 & Beijing (SIT1) & $44233345347548283534 \mathrm{I} 7 \mathrm{H} 8$ & 3 & MDR & Southern & 2009, 2010, 2012 \\
\hline 10 & Beijing (SIT1) & $44033355447548263534 \mathrm{E} 7 \mathrm{~EB}$ & 2 & QR & Eastern, Western & 2010,2011 \\
\hline 11 & Beijing (SIT1) & 44033355447548263534E7EA & 10 & MDR, QR, XDR & Northeastern, Eastern, Central, Southern & 2004, 2006-2009, 2011-2012 \\
\hline 12 & Beijing (SIT1) & $4403335544753826353477 \mathrm{GC}$ & 2 & $\mathrm{QR}, \mathrm{XDR}$ & Central, Southern & 2005,2011 \\
\hline 13 & Beijing (SIT1) & 24233354345538273634E7C9 & 2 & MDR, XDR & Central, Southern & 2008,2010 \\
\hline 14 & Beijing (SIT1) & 44232436549538283534F7CA & 2 & $\mathrm{MDR}, \mathrm{QR}$ & Northeastern & 2012 \\
\hline 15 & Beijing (SIT1) & 342133464475383A38329AB8 & 2 & QR & Central & 2009 \\
\hline 16 & Beijing (SIT1) & 34233344448539383832HA98 & 2 & QR & Northeastern & 2008,2011 \\
\hline 17 & Beijing (SIT1) & 44238245527418152 I44CA83 & 2 & QR & Northeastern & 2008,2012 \\
\hline 18 & $\begin{array}{c}\text { Manu-ancestor } \\
\text { (SIT523) }\end{array}$ & 44238246527411152I34BAB2 & 6 & XDR & Eastern, Western & $2007-2008,2012$ \\
\hline
\end{tabular}

a Order of VNTR loci: VNTRs 0424, 0577, 0580, 0802, 0960, 1644, 1955, 2136b, 2165, 2401, 2996, 3192, 3690, 4052, 4156, 1982, 2074, 2163a, 2372, 3155, 3232, 3336, 3820 and 4120. The letters in the MLVA pattern represent a copy number higher than 9; the letter A represents 10 repeats; the letter B represents 11 repeats, and so on. 


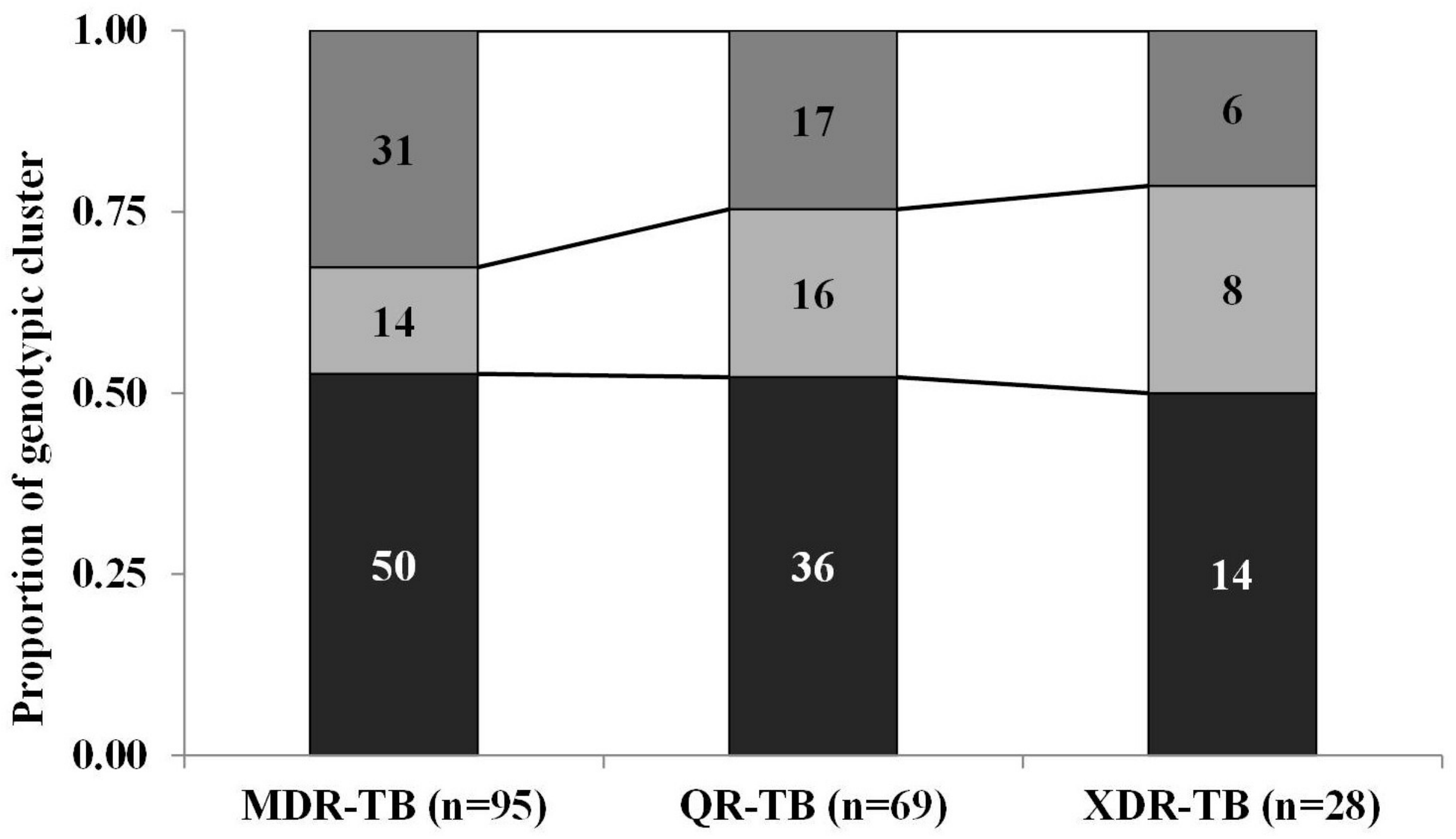

$\square$ Singletons $\square$ Minor clusters $\square$ Predominant clones 


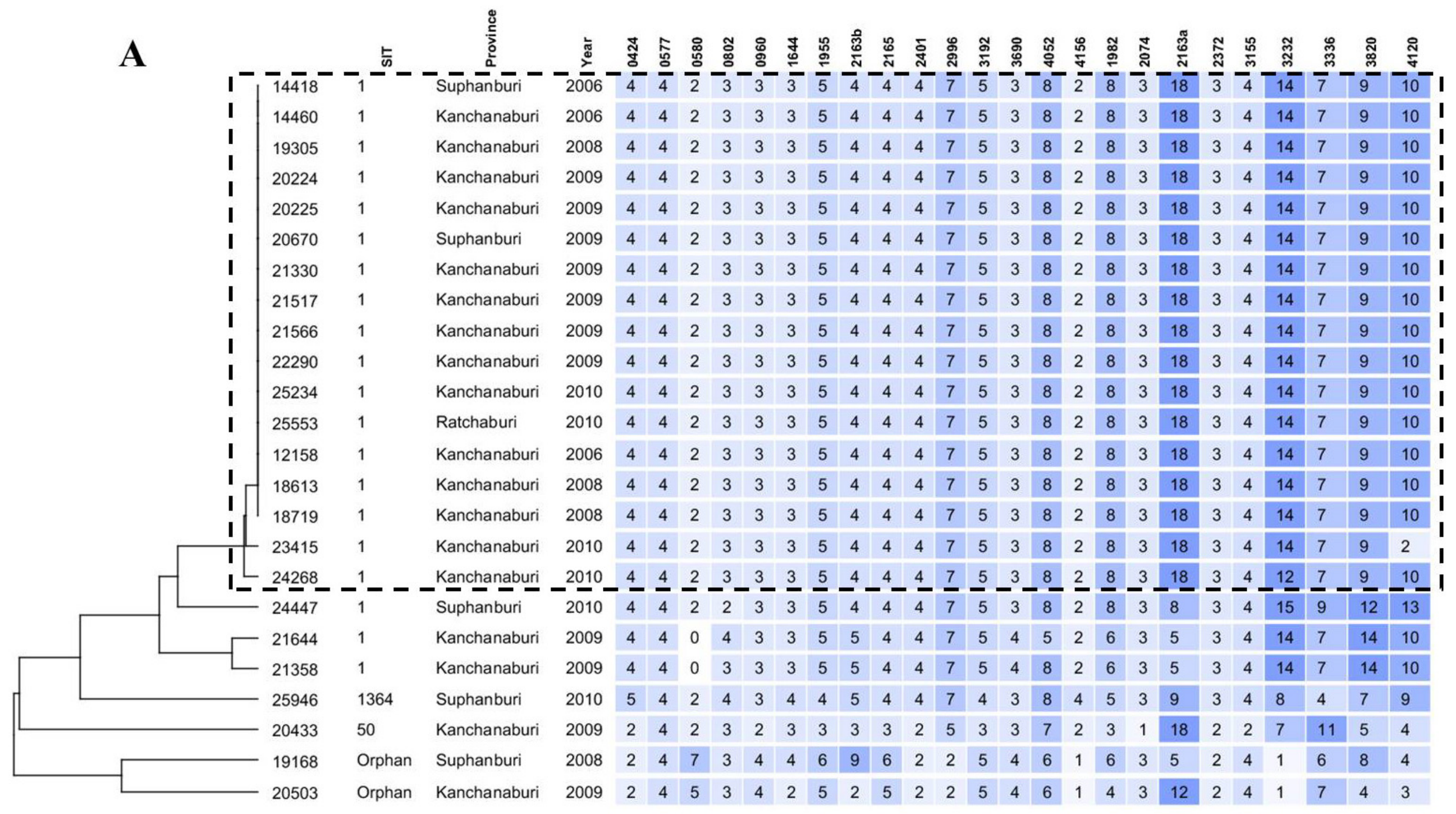




\section{B}

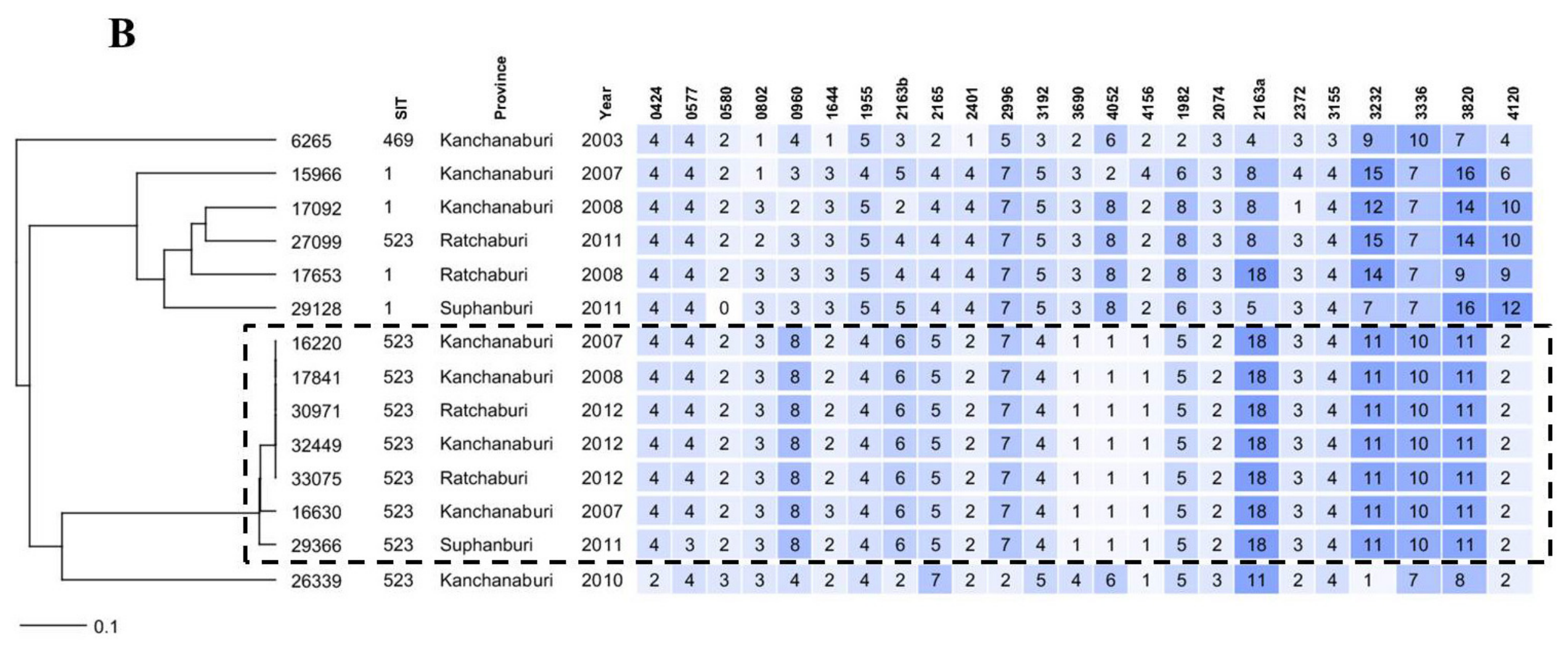


Figure $2 \mathrm{c}$

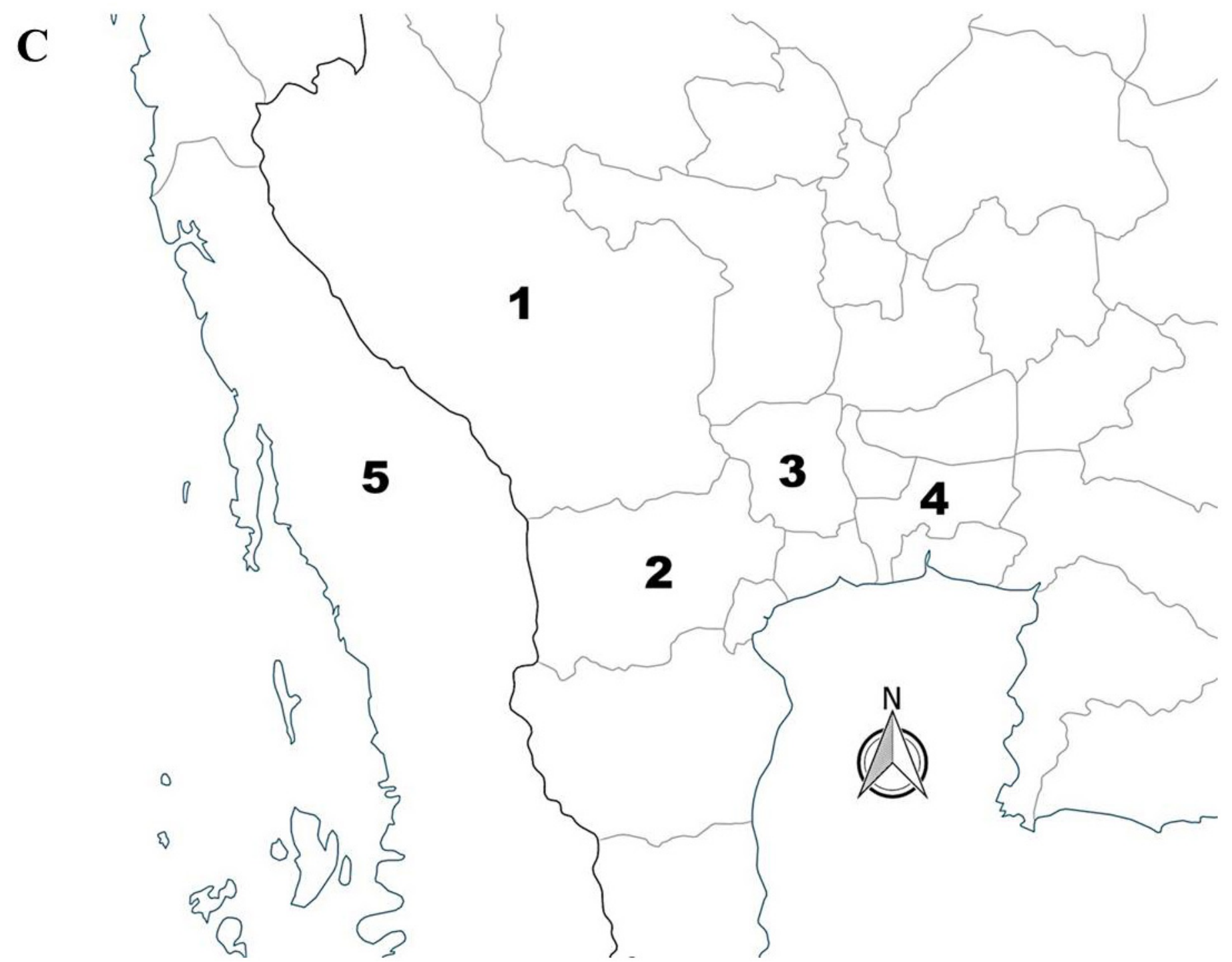

